\title{
Slat Noise Predictions using Higher-Order Finite-Difference Methods on Overset Grids
}

\author{
Jeffrey A. Housman*1 and Cetin C. Kiris ${ }^{\dagger 1}$ \\ ${ }^{1}$ NASA Ames Research Center, M/S 258-2, Moffett Field, CA 94035
}

\begin{abstract}
Computational aeroacoustic simulations using the structured overset grid approach and higher-order finite difference methods within the Launch Ascent and Vehicle Aerodynamics (LAVA) solver framework are presented for slat noise predictions. The simulations are part of a collaborative study comparing noise generation mechanisms between a conventional slat and a Krueger leading edge flap. Simulation results are compared with experimental data acquired during an aeroacoustic test in the NASA Langley Quiet Flow Facility. Details of the structured overset grid, numerical discretization, and turbulence model are provided.
\end{abstract}

\section{Introduction}

As propulsion related aircraft noise has decreased over the last decade from the use of high bypass ratio turbofan engines, a larger contribution of noise is attributed to the airframe. One of the major components of airframe noise is the leading edge high-lift device deployed during takeoff and landing. As part of NASA's Environmentally Responsible Aviation (ERA) Project, ${ }^{1}$ next generation aircraft designs are being evaluated to meet more stringent requirements on fuel burn, nitrogen oxide (NOx) emissions, and community noise. In order to design quieter leading-edge high-lift devices, a deeper understanding of the physical mechanisms which generate the noise must be examined. In this work, the noise generation mechanisms for a conventional slat are studied using high-fidelity Computational Fluid Dynamic (CFD) analysis.

The conventional slat model used in the present work is a modified version of the well known 30P30N configuration installed in the open-jet Quiet Flow Facility (QFF) at NASA Langley Research Center. The 30P30N high-lift model is a generic, unswept, 3-element, high-lift configuration with both a slat and flap deployed at 30 degrees. This configuration has been studied both experimentally ${ }^{2-5}$ and computationally. ${ }^{6-8}$ Recently, this model was used in the Benchmark problems for Airframe Noise Computations (BANC-III) workshop ${ }^{9}$ in which several teams, including the present authors, performed aeroacoustic analysis of the high-lift model in free-air. This work builds on the knowledge of previous studies by examining an installed version of the model with the flap retracted allowing the noise generation from the slat to be isolated from the noise from the flap. The present configuration has also been studied experimentally ${ }^{10,11}$ in the QFF allowing both time-averaged and unsteady surface quantities to be compared.

In Section II, the computational methodology is described, including a description of the higher-order accurate finite-difference method and the modifications to the hybrid RANS/LES turbulence model used in the analysis. The computed results are presented in Section III including the geometric model, the overset grid system, flow-field visualization, time-averaged flow quantities, and spectral data. Section IV summarizes the work.

\section{Computational Methodology}

The Launch Ascent and Vehicle Aerodynamics (LAVA) solver framework ${ }^{12}$ is utilized for the computational study. LAVA offers highly flexible meshing options and was developed with the intent of modeling highly complex geometry and flow-fields. The framework supports Cartesian and curvilinear structured grids as well as unstructured arbitrary polyhedral meshes. Overset grid technology ${ }^{13}$ is used to couple the solutions

\footnotetext{
*Research Scientist, Computational Aerosciences Branch, NAS Division, AIAA Senior Member

${ }^{\dagger}$ Branch Chief, Computational Aerosciences Branch, NAS Division, AIAA Senior Member
} 
across different overlapping meshes. In this study, the curvilinear structured overlapping grid methodology is applied. The advantage of this approach for aeroacoustic analysis of high-lift systems includes the ability to generate highly anisotropic grids to capture boundary layers, shear layers, and wakes; the high efficiency and low memory footprint of the corresponding numerical algorithm; and the straightforward and inexpensive extension to higher-order low-dissipation discretizations which are necessary for capturing flow/acoustic coupling. Allowing the structured grids to overlap each other not only simplifies the process of generating the grids (in comparison to structured multi-block abutting grids), it also allows for high quality (less-skewed) meshes, and a modular approach to adding anisotropic meshes to better resolve important flow features such as wakes in the case of high-lift models. Best practices on overset mesh generation ${ }^{14}$ for subsonic high-lift aircraft ${ }^{15}$ are expanded upon for aeroacoustic analysis of slat noise in this work. The Chimera Grid Tools $(\mathrm{CGT})^{16}$ software package is used to generate the grids, while the hole cutting and connectivity for the overset grid system is performed within the LAVA framework.

The compressible hybrid Reynolds Averaged Navier-Stokes/Large Eddy Simulation (RANS/LES) equations are solved using a finite-difference formulation applied to the non-orthogonal curvilinear transformed system of equations in strong conservation law form. ${ }^{17}$ The Spalart-Allmaras $(\mathrm{SA})^{18}$ turbulence model is used as the base RANS closure model. A zonal approach, denoted Zonal Detached Eddy Simulation (ZDES) model,${ }^{19}$ is utilized to select particular regions of the flow domain to utilize the Detached Eddy Simulation (DES) model, ${ }^{20,21}$ while other regions use the standard RANS model. Implicit second-order backward differencing is used in physical time and the discretized equations are marched in pseudo-time until a sufficient reduction in the residual has been achieved for each physical time-step (approximately 3 orders of residual reduction is achieved in the present computations). The nonlinear system of equations are linearized at each pseudo-time-step and an alternating line-Jacobi relaxation procedure is applied. Local pseudo time-stepping is used to accelerate convergence with a pseudo-time $\mathrm{CFL}=10$. Domain decomposition and the Message Passing Interface (MPI) are used to enable a scalable parallel algorithm.

\section{II.A. Higher-Order Finite-Difference Method}

Higher-order accurate finite-difference schemes have been shown to be an effective strategy for accurately and efficiently capturing noise generation phenomenon using LAVA. ${ }^{22}$ A thorough study comparing several higher-order finite-difference methods on Cartesian grids (within the LAVA framework) was reported recently. ${ }^{23}$ Results from this study indicated that higher-order Weighted Essentially Non-Oscillatory (WENO) schemes ${ }^{24}$ performed well in both resolution (Points-Per-Wavelength PPW), shock capturing, and robustness to harsh flow conditions. A natural extension of finite-difference WENO schemes to curvilinear grids are the higher-order Weighted Compact Nonlinear Schemes (WCNS). ${ }^{25}$ The WCNS method consists of WENO interpolation (as opposed to reconstruction) of left and right states to the half grid points, followed by evaluation of the numerical flux at the half points by an approximate (or exact) Riemann solver or flux vector splitting scheme, and concluding with a higher-order central finite-difference operator at the grid points which depends on the numerical fluxes at the half points in either an implicit (i.e. compact) or explicit form. When applying finite-difference methods to the curvilinear equations in strong conservation law form, standard WENO finite-difference methods will not satisfy the Geometric Conservation Law (GCL) making it necessary to combine the WENO interpolation with higher-order central-difference operators. It has been shown that free-stream preservation (i.e. the GCL condition) is satisfied up to machine precision provided that identical central difference operators are used for discretizing the metric terms as well as the convective (and divergence of the viscous) fluxes. ${ }^{26,27}$ An additional advantage of WCNS over WENO is the ability to use approximate Riemann solvers. Standard finite-difference WENO methods require the use of flux vector splitting methods for numerical flux evaluation. In this work a modified version of the Roe numerical flux is used. ${ }^{28-30}$

A side-effect of using higher-order central difference operators applied to numerical fluxes at the half grid points, which depend on higher-order WENO interpolation, is the much wider stencil required for the same order of accuracy compared to the standard finite-difference WENO method. To remove this dependency, higher-order central difference operators using a combination of the numerical fluxes at the half grid points and the physical fluxes at the grid points have been developed. ${ }^{31,32}$ This approach, denoted Hybrid Weighted Compact Nonlinear Scheme (HWCNS), allows for up to fifth-order accuracy using a seven-point stencil by combining fifth-order WENO interpolation with a sixth-order hybrid central difference operator. This is the discretization used in the present analysis and a detailed description is included in Appendix A. In addition to upwind biased WENO interpolation, a blending of central based interpolation is used to increase 
the maximum spectral resolution of the scheme. The blending factor between central and upwind biased interpolation is based on the local Mach number at the half grid point. ${ }^{33}$ This blending is only applied on selected zones which fits well with the current ZDES approach.

\section{II.B. Modifications to Hybrid RANS/LES model}

The Detached Eddy Simulation ${ }^{20,21}$ (DES) and Delayed Detached Eddy Simulation ${ }^{34-36}$ (DDES) turbulence model closures are well-tested hybrid RANS/LES models for highly separated flows. In the original DES model, the transition between RANS and LES models was based strictly on local mesh size relative to the wall-distance. For geometries with a wide range of geometric length scales, such as a high-lift device with finite-thickness leading and trailing edges, the local mesh spacing may become small enough to force transition from the RANS model to the LES model, but the mesh is typically not small enough to resolve the unsteady fluctuations causing the well-known model stress depletion. ${ }^{37}$ This brought about the modification of the model denoted DDES which attempts to maintain RANS mode in the attached boundary layer. ${ }^{34}$ Inspection of the switching function often shows a strange behavior of going from RANS near the wall, to LES, back to RANS just past the edge of the boundary layer, and subsequently back to LES. An alternative strategy appropriate for structured multi-block and overset grids is the Zonal DES (ZDES) approach ${ }^{19}$ in which specific zones are designated to use the RANS, DDES, or LES models explicitly. This is the approach taken by the present authors for modeling slat noise.

One critique of hybrid RANS/LES models is the slow development of three-dimensional turbulent structures even when the spatial and temporal resolution is more than enough to capture them. This is often caused by the definition of the local length scale in the model, which for the DDES model is the largest edge length associated with the cell (or dual cell) of a grid point. An alternative length scale definition developed using the ZDES approach ${ }^{38}$ utilizes a normalized vorticity vector together with combinations of two-dimensional length scale estimates. The normalized vorticity vector allows the span-wise direction to be identified and removes the grid length spacing in the span-wise direction (which may be large) from the local length scale estimate. This reduction is removed once three-dimensional structures are formed. At this point the normalized vorticity vector will not be aligned with any particular mesh direction and the standard length scale is recovered. Utilization of the normalized vorticity vector along with a more conservative estimate of the mesh spacing has been developed ${ }^{39}$ and is used in the present work. A detailed description of the length scale definition is included in Appendix B.

\section{Computational Aeroacoustic Analysis}

High-fidelity time-accurate simulations were performed to investigate slat noise generated by a modified version of the 30P30N high-lift model with the flap retracted. Computations of the model included open-jet installation effects to better match the conditions of the experiment. ${ }^{10}$ Preliminary analysis using unsteady RANS on both the full-span model and a two-inch centerline cross-section were performed to assess the effect of the side walls on the flow-field near the center of the model. It was found (see III.D) that the side-walls have little effect on the centerline fluid dynamics for the configuration studied in this work. Since the experiment utilizes an acoustic array which can focus on the centerline slat portion of the model, a two-inch wide cross-section was used for the aeroacoustic analysis. Similar studies have been performed for a tandem cylinder installed in the same facility with similar findings. ${ }^{40,41}$

The reference conditions used for the analysis are Mach 0.17 based on the centerline exit velocity of the open-jet and a Reynolds number of 1.7 million based on the stowed chord of 16.73 inches. Standard sea level atmospheric conditions were used for the reference pressure and temperature. A time-step of 1 microsecond was used for the time-integration of the aeroacoustic analysis and 3 orders of magnitude residual reduction of the discrete non-linear equations was enforced at each physical time-step. This equated to approximately 4 to 5 sub-iterations per physical time-step. In order to start the simulations, an unsteady RANS analysis with a larger time-step and coarser grid was run for 30 flow throughs. One flow through is defined as the time it takes for a particle exiting the nozzle, traveling at the centerline exit velocity, to pass the end of the collector plate, see Figure 1. This coarse grid solution was mapped to the finer mesh in order to warm-start the fine-grid aeroacoustic simulations. The coarse grid solutions were also used to develop specialized wake grids in the slat cove, slat wake, main element wake, and the nozzle lip regions. This enabled a locally refined mesh in regions of interest that were perfectly aligned with the time-averaged flow-field streamlines. 


\section{III.A. Geometric Model}

The geometric model used for full-span three-dimensional analysis consisted of a rectangular nozzle representing the open-jet, side walls which are attached to the nozzle and hold the high-lift model in-place, a modified (slat) $30 \mathrm{P} 30 \mathrm{~N}$ model ${ }^{42}$ with the flap retracted, and a collector plate which deflects the flow turned by the high-lift model towards a pressure exhaust vent to remove it from the anechoic chamber, see Figure 1. Modifications to the original 30P30N slat were made to the cove region, a straight section was added near the leading-edge, and the trailing-edge was thickened for installation purposes. Figure 2 (a) plots a profile of the model with the slat deployed at 30 degrees and the model rotated for an effective angle of attack of 27 degrees (based on the stowed chord line). The large black circle located at quarter chord is where the main element is mounted to the side wall for angle of attack adjustments. Large angles of attack are necessary to emulate high-lift conditions since the flap is retracted. ${ }^{43}$ Retraction of the flap is necessary in order to turn the flow less so that the collector plate effectively deflects most of the jet into the exhaust vent. The center span region of the slat and the first quarter of the main element are the areas of interest in the test. These are highlighted by red and blue coloring in Figure 2 (b) which shows the high-lift model installed between the side-walls downstream of the nozzle (which are transparent in the image).

\section{III.B. Structured Overset Grid System}

A structured overlapping grid system was generated for the installed high-lift configuration consisting of 39 zones and 900,000 grid points for a single plane of the constant span model. A span-wise width of 2 inches was used based on the work from the BANC-III workshop, ${ }^{9}$ and a total of three span-wise grid resolutions were assessed with 33, 65, and 129 grid points in the span-wise direction for a total of 30, 59, and 116 million grid points in the volume grid. The grid system was generated using the Chimera Grid Tools ${ }^{16}$ software package version $2.1 \mathrm{p}$ (CGT v2.1p). Hole-cutting and connectivity were performed by making a minimum hole-cut followed by an automated hole-offset procedure utilizing implicit hole cutting which is part of the pre-processing suite in LAVA. Triple fringe layers are enforced throughout all overlap regions to ensure proper communication between grids when using the higher-order finite-difference discretization. The computed wall $y^{+}$is less than one everywhere. The wall spacing was dictated by resolving the wall shear-stress on the upper surface of the main-element just downstream of the leading-edge. The high shear is generated by the increased velocity in the gap between the slat and main-element. A conservative stretching ratio of 1.1 is used in the wall-normal direction to ensure proper capturing of the boundary layers over each element. A more conventional stretching ratio of 1.2 is used in the stream-wise direction and uniform spacing is used in the span-wise direction.

Figure 3 shows four views of a single slice of the overset grid system. To begin the grid generation process, near-body grids for the nozzle, slat, main-element, and collector plate are hyperbolically marched from an initial discretized center-span curve representation of the geometry. O-grids are used for the main-element and collector plate, but the slat grid has a highly-concave cove region, so the boundaries of the grid in the cove were allowed to splay over each other as well as the solid surface of the slat. Once the near-body grids were complete, the off-body grid was generated as a simple stretched Cartesian grid with fine uniform spacing in the region of interest. The outer-boundaries of the off-body grid were placed 400 convective lengths (distance from the nozzle exit to the end of the collector plate) from the high-lift model center of rotation and the grid is stretched to create very large coarse cells in the far-field. The large boundary distance and coarse grid allow the acoustic waves to dissipate (due to the artificial dissipation in the numerical scheme) before reaching the outer-boundary. This minimizes the effects of pressure wave reflections. In addition to the nearbody and Cartesian off-body grids, specialized flow-aligned wake and shear-layer grids are generated for the slat, main-element, and nozzle lips. These grids are generated by hyperbolically marching from streamline curves that are generated from the time-averaged solution of a precursor calculation (which excluded these specialized grids). This allows particular flow features to be efficiently and accurately resolved since the grid is perfectly aligned to the time-averaged flow solution. Moreover, the generation of cubic cells (at least in the stream-wise and normal to the dominant shear directions) are critical to capture the three-dimensional turbulent shear-layers near the leading-edge and trailing-edge of the slat as well as the trailing edge of the main element. In the span-wise direction, the two-dimensional grid slice is repeated over the two-inch span creating a uniformly spaced grid in this direction.

Once the overlapping grids have been generated, proper hole-cutting and connectivity must be established in order to solve the governing system of equations. First, a minimal hole-cut is performed in which all grid 
points that reside inside a solid body, such as the slat, main element, nozzle, and collector plate, are identified and blanked. Next, both domain and inter-grid boundary fringe points are marked by checking if the finitedifference stencil of the discrete equations contains a blanked (i.e. a hole) point. These minimum-hole fringe points are given a very poor cell quality coefficient in order to ensure that a proper donor is found for them. Then, a cell quality for the remaining grid points (excluding physical boundary condition points) is computed based on a heuristic which combines cell volume and aspect ratio in a user-defined computational coordinate direction $(\xi, \eta$, or,$\zeta)$. This has the effect of prescribing the highest quality to both the viscous boundary layer cells and the specialized shear-layer grid cells, while giving lower quality to cells which become large in aspect ratio in non-preferred directions, as occurs on the near-body slat grid in the cove region. Now that each grid point has been marked as either a hole point, a physical boundary point, a minimum-hole fringe point, or a potential fluid point and a cell-quality has been set for all minimum hole fringe and potential fluid points, each point in the grid system searches all potential donor cells of the grid system and checks if the donor cell quality is better or not. If the potential donor cell quality is better, then the point is marked as a fringe and the points of the donor cell are marked as donor. Points which have been marked as donors do not search for potential donor cells, since these points are not allowed to become fringe points in the current implementation. The current procedure is serial because the order in which the points are visited can change the outcome of whether a point is marked as a fringe point or a donor point. If no potential donor has better quality then the current point, then the point is marked as a fluid point unless the point was originally marked as a minimum-hole fringe point, in which case it is marked as an orphan (fringe points which could not find a proper donor). The advantage of the automated hole-cutting procedure used during connectivity is clearly illustrated in Figure 3. By using the heuristic cell-quality measure based on the product of the cell-volume and a curvilinear-coordinate direction based aspect ratio, the proper mesh is retained and all other grids are blanked except in the necessary overlap regions for proper triple fringe communication between neighboring grids. This procedure enables communication between grid cells with comparable attributes with respect to the heuristic. This is very important for maintaining a continuous length scale in the hybrid RANS/LES model as well as accurate acoustic wave propagation through overset grid interfaces.

\section{III.C. Flow Field Visualization}

Simulating aeroacoustic phenomena requires high-resolution schemes which can accurately predict the acoustic generation mechanisms as well as capture linear and nonlinear wave propagation with $5-7$ points-perwavelength to be computationally feasible. Figure 4 illustrates a series of improvements made to the LAVA solver during the course of this work. In each of the sub-figures (a)-(d) an iso-contour of the Q-criteria is plotted and colored by stream-wise velocity (note the limits on the contour axis are saturated) for the medium grid with 65 points in the span-wise direction. In Figure 4 (a) the sixth-order WCNS is combined with the fifth-order upwind biased ZWENO ${ }^{44}$ interpolation along with the original DDES length scale definition. The iso-contour shows very little three-dimensional content and almost no sign of the two-dimensional Kelvin-Helmholtz instability which occurs near the leading-edge of the slat. Figure 4 (b) retains the numerical discretization but utilizes the new DDES length scale. ${ }^{39}$ Clearly the new length scale definition enables the model to capture the Kelvin-Helmholtz instability and reduces the delay in transitioning to resolved three-dimensional structures. Since the flow is subsonic everywhere, the computationally expensive and diffusive ZWENO limiters can be replaced by the optimal weight upwind-biased interpolation stencils. Elimination of the extra artificial dissipation allows more three-dimensional content to be resolved, as shown in Figure 4 (c). Higher-order interpolation and derivative operators can easily be applied in the span-wise periodic direction without requiring additional fringe points since no overlap exists in the span direction. In addition, a blending of the upwind-biased and central interpolation operators can be applied which enables even more resolvable scales on the same mesh. This is demonstrated in Figure 4 (d) which utilizes seventhorder interpolation and eighth-order central difference operators in the span-wise direction along with the blended central/upwind biased interpolation in all three coordinate directions. This is the numerical method used for the subsequent aeroacoustic results.

Visualization of the noise generating regions and sound wave propagation can be accomplished by examining the time-derivative of pressure. Figure 5 (a) shows a near-field contour plot of the pressure time-derivative on the centerline slice. Two radiating sources can be identified near the slat. The first is in the slat cove region which radiates downward and is important for estimating community noise during takeoff and landing. The second is the slat trailing-edge which radiates both forward and upward. This will affect cabin noise 
on the aircraft. The final source that can be identified is from the trailing-edge of the main-element. This source appears much smaller in magnitude than the noise sources associated with the slat region. Figure 5 (b) shows a contour plot of the time-derivative of pressure in the mid-field illustrating the propagation pattern generated from the noise sources of the high-lift device and their interaction with the open-jet shear-layers. This interaction must be well-understood to assess the validity of experimental corrections for acoustic waves passing through the shear-layer of the open-jet. ${ }^{43}$

The final flow-field visualization, Figure 6, plots instantaneous contours of the magnitude of vorticity on the centerline of the model. Large scale coherent structures representing the slat wake are seen convecting over the main-element, and shear-layers emanating from the nozzle lip are also observed. These large scale structures consist of smaller scale turbulent structures which are clearly evident in the nozzle shear-layers. The time-scale of these large scale structures is on the order of the convective velocity scale, while the small scale structures are turning at a much faster rate. The combination of these large and small scale structures are responsible for generating the acoustic waves which travel to the far-field.

\section{III.D. Comparison to Experimental Data and Grid Sensitivity Study}

High-fidelity aeroacoustic simulations require large computational resources. Before the aeroacoustic calculation was started, a series of steady and unsteady RANS calculations were performed to assess the necessary geometric elements which must be included in the aeroacoustic model. An overset grid was initially generated that included the open-jet nozzle, side-walls, full-span slat and main-element, along with the collector plate. A coarse grid resolution was used in the span-wise direction for the RANS analysis in comparison to the necessary resolution for the aeroacoustic analysis. In addition to the three-dimensional full-span grid system, a limited constant span model generated from a centerline extraction of the three-dimensional model was created, as described in sub-section III.B. This constant span model contains the same geometric components as the full-span model with the exception of the side-walls. Figure 7 plots a comparison of the time-averaged $\mathrm{Cp}$ for the experiment, the constant span (2D) model, and the full-span (3D) model including side walls. The Cp curves for both the 3D and 2D models are almost on top of each other. There is a small difference on the suction-side of the slat shown in Figure 7 (b) likely caused by side wall effects. When comparing the computational results to the experiment, an over-prediction of both suction-side and pressure-side $\mathrm{Cp}$ on the slat is observed. This suggests that the installed angle of attack may need to be adjusted for the computation. Using the constant span model the angle of attack was reduced from 27 degrees to 26 degrees. Figure 8 plots the Cp comparison between the experiment and the computational model at both 26 and 27 degrees. The Cp profiles between the experiment and the 26 degree computational results fall almost on top of each other. The adjusted angle of attack of 26 degrees was used for the subsequent aeroacoustic analysis.

The stream-wise and wall-normal grid resolution used for the unsteady RANS analysis appear to be sufficient based on the time-averaged Cp comparison, but the span-wise grid spacing must be assessed for the aeroacoustic analysis. Figures 9 (a)-(c) plot iso-contours of Q-criteria colored by stream-wise velocity using the coarse, medium, and fine span-wise grids. As the span-wise resolution increases, the length over which the two-dimensional Kelvin-Helmholtz instability develops into three-dimensional turbulent structures decreases. This effectively increases the resolved turbulent kinetic energy in the slat cove region which is important for capturing noise generation. Figure 9 (d) plots the layout for the Kulite dynamic pressure transducers on the slat and main element where PSD spectral analysis is performed.

The predicted PSD spectrum on the slat at dynamic pressure transducers 1, 2, 3, and 4 using each of the three span-wise mesh resolutions is compared to the experimental data in Figures 10 (a)-(d). A constant shift down in PSD magnitude (approx. 10.84dB) related to half the frequency bin width, $12.2 \mathrm{~Hz}$ has been applied to all the experimental data. The necessity of the shift is currently under investigation by the QFF test-team. At dynamic pressure transducer locations 1 and 3 the PSD spectrum between the 65 and 129 span-wise grid point meshes are nearly on top of each other, while the coarser mesh shows a faster decay at higher frequencies. This is consistent with the reduction in predicted turbulent kinetic energy on the coarse grid, demonstrated in the previous figure. Excellent agreement in broadband content with the shifted experimental data is observed at locations 1,2 , and 3 over the entire frequency range of interest. Dynamic pressure transducer 4 shows a different trend in which the coarse, medium, and fine grid spectra are very close up to a frequency of $4 \mathrm{kHz}$ and after $6 \mathrm{kHz}$, but the finest grid predicts a much higher PSD than the coarse and medium grids in the $4-6 \mathrm{kHz}$ band. Comparing the CFD prediction to the experimental data at location 4 shows an over-prediction of the PSD by the CFD when compared to experiment. This may be caused by an under-resolved mesh deep in the cove region, or a suppression of the three-dimensional turbulent structures 
caused by specifying the boundary layer zones to be in RANS mode. It is also observed that locations 2 and 4 show a high-frequency peak in the spectrum of the CFD result at around $6.5-7 \mathrm{kHz}$ which is associated with the finite-thickness trailing-edge of the slat. The experimental data show several peaks at much lower frequencies. After discussing the computation model with the experimental team, it was mentioned that the as-built slat has a thicker trailing edge than the CAD model used for the computational analysis which was performed several months before the test. A thicker trailing edge will certainly decrease the shedding frequency, and other facility effects not modeled in the computational analysis may be responsible for the peaky behavior of the experimental data. ${ }^{10}$

The PSD spectra on the main element at dynamic pressure transducers 6, 7, 9, and 10 are compared in Figure 11 (a)-(d). The same shift has been applied to the experimental data, as done for the slat comparison. The comparison between CFD and experiment at location 6 show a large under-prediction by the CFD. This is caused by the increased stream-wise spacing of the mesh over the main-element which is too coarse to resolve the three-dimensional structures convecting downstream of the slat-gap. Location 7 shows some improvement at the higher-frequencies, but also shows the curious behavior that the finer span-wise grid predicts about a $3 \mathrm{~dB}$ reduction in PSD across the frequency range compared to the coarse and medium grids. Since the stream-wise spacing is still too large here for accurate convection, no strong conclusion can be drawn here. Good agreement of the broadband content is achieved at locations 9 and 10 near the leading edge of the main-element and positioned 1 inch apart from each other in the span-wise direction. In this region the mesh is highly resolved and the medium and fine grid results are nearly on top of each other.

From the PSD comparisons on the slat and main-element (in the resolved region) it appears that the medium grid is sufficient for the present analysis. Figure 12 (a) plots the PSD spectrum for all the dynamic pressure transducers on the slat from the medium grid solution. A much larger frequency range is plotted here showing the fast drop-off in PSD when the frequency becomes too large relative the mesh spacing and resolution capacity of the numerical method. Dynamic pressure transducers 1 and 3 are located where the wake from the slat leading-edge impinges on the lower surface of the slat. This is a dominant source of the broadband noise which is clearly visible in the spectrum, and all tonal content at these locations are completely covered by the large magnitude of the broadband noise. The remaining dynamic pressure transducers on the slat show several tonal peaks starting at approximately $6.5-7 \mathrm{kHz}$ and show large magnitudes of broadband noise at lower frequencies. The PSD spectrum for the dynamic pressure transducers located on the main-element are shown in Figure 12 (b). Tonal content is observed for each of the dynamic pressure transducers again starting at $6.5-7 \mathrm{kHz}$ as observed on the slat. The tones are much more pronounced in the $20-40 \mathrm{kHz}$ range on the main-element when compared to the slat. As already mentioned, these tones are likely caused by shedding from the finite-thickness trailing-edge of the slat which is much larger relative to the slat chord in the model than what would typically be found on and full-size aircraft wing. Dynamic pressure transducers 9 and 10 which are located at the same stream-wise location but off-set from each other by one inch in the span-wise direction show almost identical spectrum.

\section{Summary}

The Launch Ascent and Vehicle Aerodynamics (LAVA) solver framework using structured curvilinear overlapping grids has been successfully applied to the prediction of slat noise for a high-lift model installed in the QFF open-jet facility. A detailed description of the numerical method, turbulence model, high-lift and QFF facility model geometry, and structured overset grid system has been presented. Demonstration of the solver improvements in convective flux discretization, hybrid RANS/LES turbulence modeling, and implicit hole-cutting/connectivity have been outlined. Unsteady RANS analysis was performed on a full-span configuration of the model including side-walls, as well as a mid-span extracted configuration. Comparison of time-averaged $\mathrm{Cp}$ from the experiment and the two grid systems indicate that the constant span model is sufficient for the aeroacoustic analysis. In addition, an adjustment of the installed angle of attack by one degree provided a significant improvement in the time-averaged $\mathrm{Cp}$ comparison with experimental data, and was used for the aeroacoustic analysis.

High-fidelity time-accurate aeroacoustic analysis was performed on three grid systems with varying resolutions in the span-wise direction. A sensitivity analysis was performed for the surface PSD spectrum indicating that the medium span-wise grid resolution was sufficient for aeroacoustic analysis. Comparison of the PSD spectrum with experimental data showed excellent agreement in locations where the mesh was well-resolved, while the agreement became worse with distance along the main-element caused by coarsening 
of the stream-wise grid resolution in this region. Flow visualization of the time-derivative of pressure identified three noise source generation areas: the impingement of the slat leading edge wake onto the slat-surface in the cove region, the trailing-edge of the slat, and the trailing-edge of the main-element. Analyzing the PSD spectrum indicates that the impingement source is the dominant broadband noise source, while high frequency tones are likely a consequence of the finite-thickness trailing-edge of the slat.

\section{Acknowledgements}

The work was funded by the NASA Environmentally Responsible Aviation (ERA) Project. The authors would like to thank Fayette Collier, Russell Thomas, and Kevin James for their support. The authors also wish to acknowledge the efforts of Christopher Bahr and Florence Hutcheson, and Casey Burley of NASA Langley for the efforts in the experimental analysis of the configuration and several discussions regarding the test facility. Additionally, we thank H. Dogus Akaydin for his efforts on the Krueger design portion of the project, which will be evaluated using the computational aeracoustic analysis outlined in the work in the future, Yueping Guo for invaluable insight in the subject of slat noise generation. Computer time has been provided by the NASA Advanced Supercomputing (NAS) facility at NASA Ames Research Center.

\section{References}

${ }^{1}$ Collier, F., "Environmentally Responsible Aviation (ERA) Project," NASA Fundamental Aeronautics Program, Third Annual Meeting, September 29 - October 1 2009, presentation.

${ }^{2}$ Paschal, K., Jenkins, L., and Yao, C., "Unsteady Slat-Wake Characteristics of a High-Lift Configuration," 38th Aerospace Sciences Meeting, Reno, Nevada, January 2000, AIAA-2000-0139.

${ }^{3}$ Jenkins, L., Khorrami, M., and Choudhari, M., "Characterization of Unsteady Flow Structures Near Leading-Edge Slat: Part I. PIV Measurements," 10th AIAA/CEAS Aeroacoustics Conference, Manchester, Great Britain, May 2004, AIAA-20042801.

${ }^{4}$ Murayama, M., Nakakita, K., Yamamoto, K., Ura, H., and Ito, Y., "Experimental Study of Slat Noise from 30P30N Three-Element High-Lift Airfoil in JAXA Hard-Wall Low-Speed Wind Tunnel," 20th AIAA/CEAS Aeroacoustics Conference, June 2014, AIAA-2014-2080.

${ }^{5}$ Pascioni, K. A., Cattafesta, L. N., and Choudhari, M. M., "An Experimental Investigation of the 30P30N Multi-Element High-Lift Airfoil," 20th AIAA/CEAS Aeroacoustics Conference, June 2014, AIAA-2014-3062.

${ }^{6}$ Khorrami, M., Choudhari, M., and Jenkins, L., "Characterization of Unsteady Flow Structures Near Leading-Edge Slat: Part II. 2D Computations," 10th AIAA/CEAS Aeroacoustics Conference, Manchester, Great Britain, May 2004, AIAA-20042802.

${ }^{7}$ Choudhari, M. and Khorrami, M., "Effect of Three-Dimensional Shear-Layer Structures on Slat Cove Unsteadiness," AIAA Journal, Vol. 45, No. 9, 2007, pp. 2174-2186.

${ }^{8}$ Lockard, D. and Choudhari, M., "Noise Radiation from a Leading-Edge Slat," 15th AIAA/CEAS Aeroacoustics Conference, May 2009, AIAA-2009-3101.

${ }^{9}$ Choudhari, M. and Lockard, D. P., "Assessment of Slat Noise Predictions for 30P30N High-Lift Configuration from BANC-III workshop," 21st AIAA/CEAS Aeroacoustics Conference, Dallas, Texas, June 2015, AIAA-2015-3139.

${ }^{10}$ Bahr, C., Hutcheson, F., Thomas, R., and Housman, J., "A Comparison of the Noise Characteristics of a Conventional Slat and Krueger Flap," 22nd AIAA/CEAS Aeroacoustics Conference, Lyon, France, May 2016, accepted.

${ }^{11}$ Akaydin, H. D., Housman, J., Kiris, C., Bahr, C., and Hutcheson, F., "Krueger flaps revisited: a computational aerodynamic and aeroacoustic design study," 22nd AIAA/CEAS Aeroacoustics Conference, Lyon, France, May 2016, accepted.

${ }^{12}$ Kiris, C., Barad, M., Housman, J., Sozer, E., Brehm, C., and Moini-Yekta, S., "The LAVA Computational Fluid Dynamics Solver," 52st AIAA Aerospace Sciences Meeting, National Harbor, Maryland, January 13-17, 2014, AIAA-2014-0070.

${ }^{13}$ Steger, J. L. and Benek, J. A., "On the Use of Composite Grid Schemes in Computational Aerodynamics," Technical Memorandum 88372, NASA, 1986.

${ }^{14}$ Chan, W., Gomez, R. J., Rogers, S., and Buning, P., "Best Practices in Overset Grid Generation," 32nd AIAA Fluid Dynamics Conference and Exhibit, St. Louis, Missouri, June 24-26, 2002, AIAA-2002-3191.

${ }^{15}$ Rogers, S. E., Roth, K. R., M., N. S., Baker, M. D., Slotnick, J. P., Whitlock, M., and Cao, H. V., "Advances in Overset CFD Processes Applied to Subsonic High-Lift Aircraft," 18th AIAA Applied Aerodynamics Conference, Denver, CO, 2000, AIAA-2000-4216.

${ }^{16}$ Chan, W., "Developments in Strategies and Software Tools for Overset Structured Grid Generation and Connectivity," 20th AIAA Computational Fluid Dynamics Conference, Honolulu, Hawaii, June 2011, AIAA-2011-3051.

${ }^{17}$ Vinokur, M., "Conservation Equations of Gasdynamics in Curvilinear Coordinate Systems," Journal of Computational Physics, Vol. 14, 1974, pp. 105-125.

${ }^{18}$ Spalart, S. R. and Allmaras, S. A., "A One-Equation Turbulence Model for Aerodynamic Flows," 30th Aerospace Sciences Meeting and Exhibit, Reno, NV, January 1992, AIAA-92-0439.

${ }^{19}$ Deck, S., "Recent improvements in the zonal detached-eddy simulation (ZDES) formulation," Theoretical and Computational Fluid Dynamics, Vol. 26, 2012, pp. 523-550.

${ }^{20}$ Spalart, S. R., Jou, W.-H., Strelets, M., and Allmaras, S. R., "Comments on the feasibility of LES for wings, and on a hybrid RANS/LES approach," First AFOSR international conference on DNS/LES, Ruston, Louisiana, August 1997, Greyden Press. 
${ }^{21}$ Strelets, M., "Detached eddy simulation of massively separated flows," 39th Aerospace Sciences Meeting and Exhibit, Reno, Nevada, January 2001, AIAA-2001-0879.

${ }^{22}$ Brehm, C., Housman, J., and Kiris, C., "Noise Generation Mechanisms for a Supersonic Jet Impinging on an Inclined Plate," Journal of Fluid Mechanics, 2015, In review.

${ }^{23}$ Brehm, C., Barad, M., Housman, J., and Kiris, C., "A comparison of higher-order finite-difference shock capturing schemes," Computers and Fluids, Vol. 122, November 2015, pp. 184-208.

${ }^{24}$ Shu, C.-W., "High Order Weighted Essentially Nonoscillatory Schemes for Convection Dominated Problems," SIAM Review, Vol. 51, No. 1, 2009, pp. 92-126.

${ }^{25}$ Deng, X. and H., Z., "Developing High-Order Weighted Compact Nonlinear Schemes," Journal of Computational Physics, Vol. 165, 2000, pp. 22-44.

${ }^{26}$ Vinokur, M. and Yee, H., "Extension of Efficient Low Dissipation High Order Schemes for 3-D Curvilinear Moving Grids," Frontiers of Computational Fluid Dynamics, World Scientific, 2002, pp. 129-163, edited by D. A. Caughey and M. M. Hafez.

${ }^{27}$ Deng, X., Mao, M., Tu, G., Liu, H., and Zhang, H., "Geometric conservation law and applications to high-order finite difference schemes with stationary grids," Journal of Computational Physics, Vol. 230, 2011, pp. 1100-1115.

${ }^{28}$ Housman, J., Kiris, C., and Hafez, M., "Preconditioned methods for simulations of low speed compressible flows," Computes and Fluids, Vol. 38, No. 7, August 2009, pp. 1411-1423.

${ }^{29}$ Housman, J., Kiris, C., and Hafez, M., "Time-Derivative Preconditioning Methods for Multicomponent Flows - Part I: Riemann Problems," Journal of Applied Mechanics, Vol. 76, No. 2, February 2009.

${ }^{30}$ Housman, J., Kiris, C., and Hafez, M., "Time-Derivative Preconditioning Methods for Multicomponent Flows - Part II: Two-Dimensional Applications," Journal of Applied Mechanics, Vol. 76, No. 3, March 2009.

${ }^{31}$ Deng, X., Mao, M., Jiang, Y., and Liu, H., "New High-Order Hybrid Cell-Edge and Cell-Node Weighted Compact Nonlinear Schemes," 20th AIAA Computational Fluid Dynamics Conference, Honolulu, Hawaii, June 2011, AIAA-2011-3857.

${ }^{32}$ Nonomura, T. and Fujii, K., "Robust explicit formulation of weighted compact nonlinear scheme," Computers 8 Fluids, Vol. 85, 2013, pp. 8-18.

${ }^{33}$ Thornber, B., Mosedale, A., Drikakis, D., Youngs, D., and Williams, R. J., "An improved reconstruction method for compressible flow with low Mach number features," Theoretical and Computational Fluid Dynamics, Vol. 26, 2012 , pp. 523-550.

${ }^{34}$ Spalart, P. R., Deck, S., Shur, M. L., Squires, K. D., M., S., and Travin, A., "A new version of detached-eddy simulation, resistant to ambiguous grid densities," Theoretical and Computational Fluid Dynamics, Vol. 20, 2006, pp. 181-195.

${ }^{35}$ Shur, M., Spalart, P. R., Strelets, M., and Travin, A., "A hybrid RANS-LES approach with delayed-DES and wallmodelled LES capabilities," International Journal of Heat and Fluid Flow, Vol. 29, 2008, pp. 1638-1649.

${ }^{36}$ Spalart, P. R., "Detached-Eddy Simulation," Annual Review Fluid Mechanics, Vol. 41, 2009, pp. 181-202.

${ }^{37}$ Caruelle, B. and Ducros, F., "Detached-Eddy Simulations of Attached and Detached Boundary Layers," International Journal of Computational Fluid Dynamics, Vol. 17, 2003, pp. 433-451.

${ }^{38}$ Chauvet, N., Deck, S., and Jacquin, L., "Zonal detached eddy simulation of a controlled propulsive jet," AIAA Journal, Vol. 45, No. 10, 2007, pp. 2458-2473.

${ }^{39}$ Shur, M., Spalart, P. R., Strelets, M., and Travin, A., "An enhanced version of DES with rapid transition from RANS to LES in separated flows," Flow, Turbulence and Combustion, Vol. submitted, 2015.

${ }^{40}$ Bres, G. A., "Flow and Noise Predictions for Tandem Cylinders in a Realistic Wind-Tunnel Configuration," 17th AIAA/CEAS Aeroacoustics Conference (32nd AIAA Aeroacoustics Conference), Portland, Oregon, June 2011, AIAA-20112824.

${ }^{41}$ Greschner, B., Eschricht, D., Mockett, C., and F., T., "Turbulence Modelling effects on Tandem Cylinder Interaction Flow and Analysis of Installation effects on broadband noise using Chimera Technique," 30th AIAA Applied Aerodynamics Conference, New Orleans, Louisiana, June 2012, AIAA-2012-3033.

${ }^{42}$ Turner, T., Kidd, R., Hartl, D., and Scholten, W., "Development of a SMA-Based, Slat-Cove Filler for Reduction of Aeroacoustic Noise Associated with Transport-Class Aircraft Wings," Proceedings of the ASME 2013 Conference on Smart Materials, Adaptive Structures and Intelligent Systems, Snowbird, UT, 2013.

${ }^{43}$ Mendoza, J., Brooks, T., and Humphreys, W. M., "Aeroacoustic Measurements of a Wing/Slat Model," 8th AIAA/CEAS Aeroacoustics Conference, Breckenridge, Colorado, June 2002, AIAA-2002-2604.

${ }^{44}$ Borges, R., Carmona, M., Costa, B., and Don, W., "An improved weighted essentially non-oscillatory scheme for hyperbolic conservation laws," Journal of Computational Physics, Vol. 227, No. 6, 2008, pp. 3191-3211.

${ }^{45}$ Breuer, M., Jovicic, N., and Mazaev, K., "Comparison of DES, RANS, and LES for the Separated Flow Around a Flat Plate at High Incidence," International Journal for Numerical Methods in Fluids, Vol. 41, 2003, pp. 357-388.

${ }^{46}$ Deck, S., "Zonal detached eddy simulation of the Flow Around a High-Lift Configuration," AIAA Journal, Vol. 43, No. 11, 2005, pp. 2372-2384.

${ }^{47}$ Ashton, N., Prosser, R., and Revell, A., "A hybrid numerical scheme for a new formulation of delayed detached-eddy simulation (DDES) based on elliptic relaxation," Journal of Physics: Conference Series, Vol. 318, dec 2011, p. 042043. 


\section{Appendix A: Hybrid Weighted Compact Nonlinear Scheme}

The Hybrid Weighted Compact Nonlinear Scheme implemented in the LAVA structured curvilinear overset grid solver utilizes an explicit sixth-order central differencing operator based on a combination of numerical fluxes at the half grid-points and physical fluxes at the grid points. The approximation to the convective flux derivative in the $\xi$ direction is,

$$
\frac{\partial \hat{F}}{\partial \xi} \approx \frac{1}{\Delta \xi}\left[a_{1}\left(\tilde{F}_{j+1 / 2}-\tilde{F}_{j-1 / 2}\right)+a_{2}\left(\hat{F}_{j+1}-\hat{F}_{j-1}\right)+a_{3}\left(\hat{F}_{j+2}-\hat{F}_{j-2}\right)\right]
$$

where $a_{1}=\frac{64}{45}, a_{2}=\frac{-2}{9}$, and $a_{3}=\frac{1}{180}$. Near the boundary at $j=2$ and $j=J M A X-1$ a fourth-order central differencing operator is used,

$$
\frac{\partial \hat{F}}{\partial \xi} \approx \frac{1}{\Delta \xi}\left[b_{1}\left(\tilde{F}_{j+1 / 2}-\tilde{F}_{j-1 / 2}\right)+b_{2}\left(\hat{F}_{j+1}-\hat{F}_{j-1}\right)\right]
$$

where $b_{1}=\frac{4}{3}$ and $b_{2}=\frac{-1}{6}$. The solution at the boundary nodes is determined by boundary condition operators. The physical flux at the grid points is denoted by $\hat{F}_{j}$ and is simply evaluated using the current solution at the grid points. The numerical flux, denoted by $\tilde{F}_{j+1 / 2}$, is evaluated at the half grid points using the modified Roe scheme,

$$
\tilde{F}_{j+1 / 2}\left(Q_{L}, Q_{R}\right)=\frac{1}{2}\left[\hat{F}\left(Q_{R}\right)+\hat{F}\left(Q_{L}\right)-\Gamma_{p}\left|\Gamma_{p}^{-1} \hat{A}(\bar{Q})\right|\left(Q_{R}-Q_{L}\right)\right] .
$$

Here $Q_{L}$ and $Q_{R}$ are the left and right upwind biased interpolations of the primitive variables $Q=$ $(p, u, v, w, T)^{T}$, where $p$ is the pressure, $(u, v, w)$ are the Cartesian velocity components, and $T$ is the temperature. The artificial dissipation matrix $\Gamma_{p}\left|\Gamma_{p}^{-1} \hat{A}\right|$ is evaluated at the density-weighted averaged state,

$$
\bar{Q}=\left[\begin{array}{c}
\sqrt{\rho_{L}} p_{L}+\sqrt{\rho_{R}} p_{R} \\
\sqrt{\rho_{L}} u_{L}+\sqrt{\rho_{R}} u_{R} \\
\sqrt{\rho_{L}} v_{L}+\sqrt{\rho_{R}} v_{R} \\
\sqrt{\rho_{L}} w_{L}+\sqrt{\rho_{R}} w_{R} \\
\sqrt{\rho_{L}} T_{L}+\sqrt{\rho_{R}} T_{R}
\end{array}\right] .
$$

Details on the preconditioning matrix, $\Gamma_{p}$, and the preconditioned flux Jacobian matrix, $\Gamma_{p}^{-1} \hat{A}$, can be found in Housman et al. ${ }^{28-30}$ The fifth-order left and right biased primitive variable interpolations to the half grid point are given by,

$$
\begin{aligned}
& Q_{L}=c_{1} Q_{j-2}+c_{2} Q_{j-1}+c_{3} Q_{j}+c_{4} Q_{j+1}+c_{5} Q_{j+2} \\
& Q_{R}=c_{5} Q_{j-1}+c_{4} Q_{j}+c_{3} Q_{j+1}+c_{2} Q_{j+2}+c_{1} Q_{j+3},
\end{aligned}
$$

where $c_{1}=\frac{3}{128}, c_{2}=-\frac{5}{32}, c_{3}=\frac{45}{64}, c_{4}=\frac{15}{32}$, and $c_{5}=-\frac{5}{128}$. For seven-order accurate interpolations,

$$
\begin{aligned}
& Q_{L}=d_{1} Q_{j-3}+d_{2} Q_{j-2}+d_{3} Q_{j-1}+d_{4} Q_{j}+d_{5} Q_{j+1}+d_{6} Q_{j+2}+d_{7} Q_{j+3} \\
& Q_{R}=d_{7} Q_{j-2}+d_{6} Q_{j-1}+d_{5} Q_{j}+d_{4} Q_{j+1}+d_{3} Q_{j+2}+d_{2} Q_{j+3}+d_{1} Q_{j+4},
\end{aligned}
$$

where $d_{1}=-\frac{5}{1024}, d_{2}=\frac{21}{512}, d_{3}=-\frac{175}{1024}, d_{4}=\frac{175}{256}, d_{5}=\frac{525}{1024}, d_{6}=-\frac{35}{512}, d_{7}=\frac{7}{1024}$.

To increase the spectral resolution of the scheme a blending of centered and biased interpolations of the left and right states of the velocity components is performed. The blending is dependent on the local Mach number and is given by,

$$
\begin{aligned}
& \vec{U}_{L}=\frac{1}{2}\left(\vec{U}_{L}+\vec{U}_{R}\right)+\frac{\psi}{2}\left(\vec{U}_{L}-\vec{U}_{R}\right) \\
& \vec{U}_{L}=\frac{1}{2}\left(\vec{U}_{L}+\vec{U}_{R}\right)+\frac{\psi}{2}\left(\vec{U}_{R}-\vec{U}_{L}\right),
\end{aligned}
$$

where $\psi=\min \left(\max \left(M_{L}, M_{R}, 0\right), 1\right)$ and $M_{L}$ and $M_{R}$ are the Mach numbers computed using the biased left and right state variables. When $\psi=1$ the biased interpolations are recovered. For $\psi=0$, a sixthorder centered interpolation is formed from the fifth-order biased interpolations, and an eight-order centered 
interpolation is formed from the seventh-order biased interpolations. The blended scheme is only used in selected zones, typically in zones where the DES model is in LES mode and the grid is relatively uniform. Spurious oscillations have been observed when utilizing the blending procedure on highly stretched grids, such as boundary layer grids, and is avoided. In addition to changing the length scale in the model the near-wall functions are removed in LES mode. ${ }^{45,46}$

\section{Appendix B: Zonal Delayed Detached Eddy Simulation Model}

The hybrid RANS/LES model used in the aeroacoustic analysis is a Zonal Detached Eddy Simulation model. The baseline RANS model is the Standard Spalart-Allmaras One-Equation Model ${ }^{18}$ as described on the "NASA Turbulence Modeling Resource" (the equations are not repeated here for brevity). The model is transformed to curvilinear coordinates and solved in non-conservative form using a first-order accurate upwind discretization for the convective terms, second-order central differencing for the viscous terms, and second-order backward differencing in physical time. As with the Navier-Stokes equations, a dual-time procedure is utilized where a psuedo-time derivative is added to the equations and discretized using a first-order backward differecing. The turbulence model equation is solved decoupled and updated at each pseudo-time step until both the field equations and the turbulence model equation are converged (three oders of magnitude residual reduction for the field equations and two orders for the turbulence model in the current analysis). Zones which are prescribed by the user to be in URANS mode are solved with the baseline model. The zones which are prescribed to be in DES mode utilize a modified length scale instead of the wall-distance in the original SA RANS model,

$$
d=\left\{\begin{array}{ccc}
d_{\mathrm{wall}} & \text { if } & d_{\mathrm{wall}}<C_{\mathrm{DES}} \Delta_{s} \\
C_{\mathrm{DES}} \Delta_{s} & & \text { otherwise. }
\end{array}\right.
$$

The $C_{D E S}$ parameter is set to 0.6 based on matching the decay rate for homogenous isotropic turbulence. ${ }^{47}$ The local grid length scale, $\Delta_{s}$ is defined as,

$$
\Delta_{s}=\max \left(\Delta_{s j}, \Delta_{s k}, \Delta_{s l}\right) / \sqrt{3}
$$

The $\Delta_{s i}$ for $i=j, k, l$ are given by,

$$
\Delta_{s i}=\sqrt{\left(n_{y} \Delta_{i z}-n_{z} \Delta_{i y}\right)^{2}+\left(n_{z} \Delta_{i x}-n_{x} \Delta_{i z}\right)^{2}+\left(n_{x} \Delta_{i y}-n_{y} \Delta_{i x}\right)^{2}} .
$$

Now

$$
\begin{aligned}
\Delta_{i x} & =\frac{1}{2}\left(x_{i+1}-x_{i-1}\right) \\
\Delta_{i y} & =\frac{1}{2}\left(y_{i+1}-y_{i-1}\right) \\
\Delta_{i z} & =\frac{1}{2}\left(z_{i+1}-z_{i-1}\right)
\end{aligned}
$$

and

$$
\begin{aligned}
& n_{x}=\frac{\Omega_{x}^{*}}{\left\|\Omega^{*}\right\|} \\
& n_{y}=\frac{\Omega_{y}^{*}}{\left\|\Omega^{*}\right\|} \\
& n_{z}=\frac{\Omega_{z}^{*}}{\left\|\Omega^{*}\right\|} .
\end{aligned}
$$

In order to avoid division by zero in a constant flow region to appropriately scale the normalized vorticity vector, $\vec{n}$, the scaled vorticity vector, $\vec{\Omega}^{*}=\vec{\Omega}+\vec{\epsilon}$ where $\vec{\epsilon}=10^{-8} \vec{x} / \sqrt{3}$, as described in Shur et al. ${ }^{39}$ This procedure has the effect of reducing the influence of the grid spacing in the direction of the vorticity vector, thus for a typical two-dimensional vortex roll-up in the wake, the span-wise grid spacing is ignored in the modified length scale until three-dimensional structures are generated. At that point the normalized vorticity vector will not be directly aligned with a particular grid coordinate direction and the standard local length scale is recovered. 

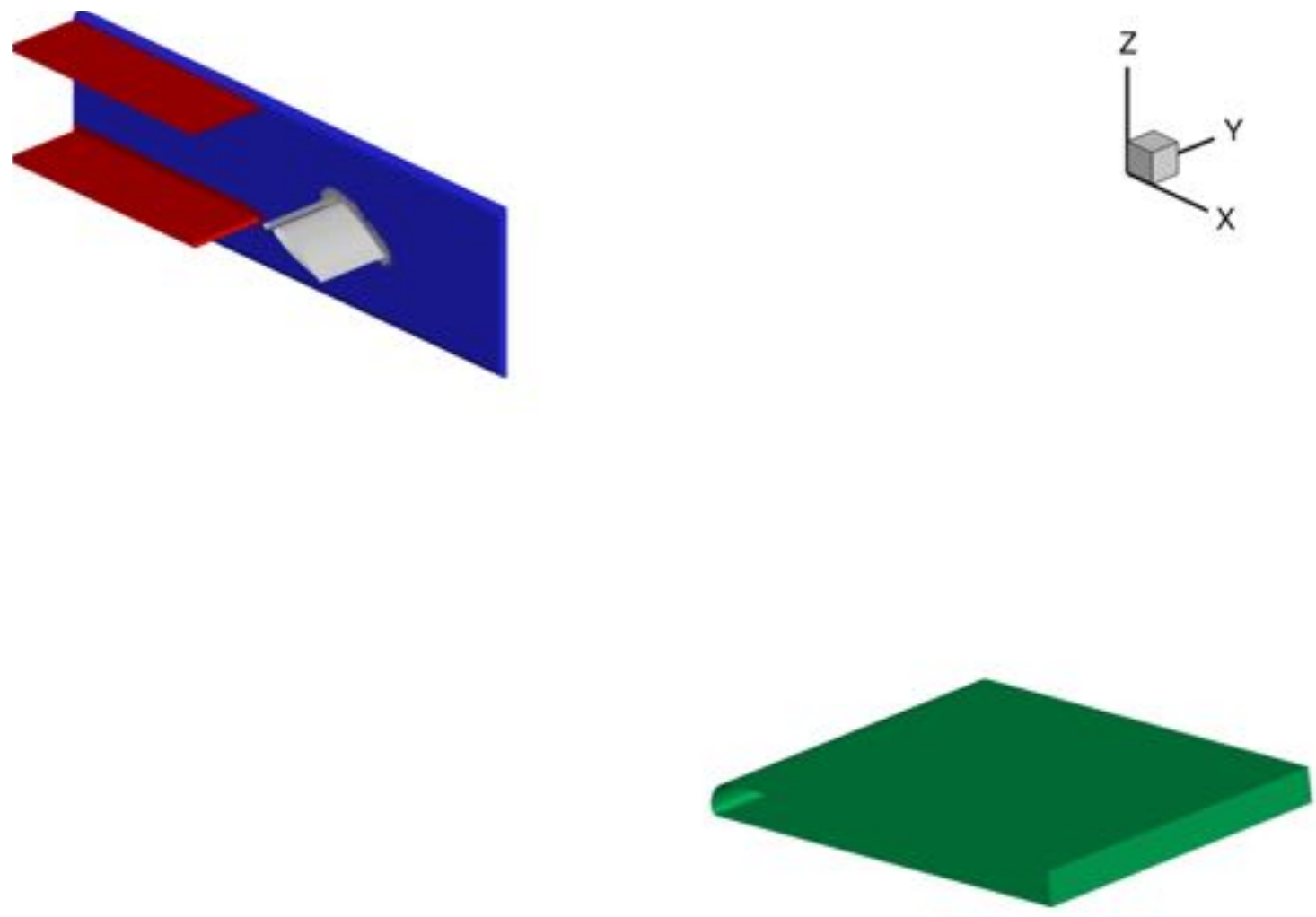

Figure 1. Half-body isometric CAD view of the simplified nozzle colored red, the side-wall colored blue, the installed high-lift model colored grey, and the collector plate colored green

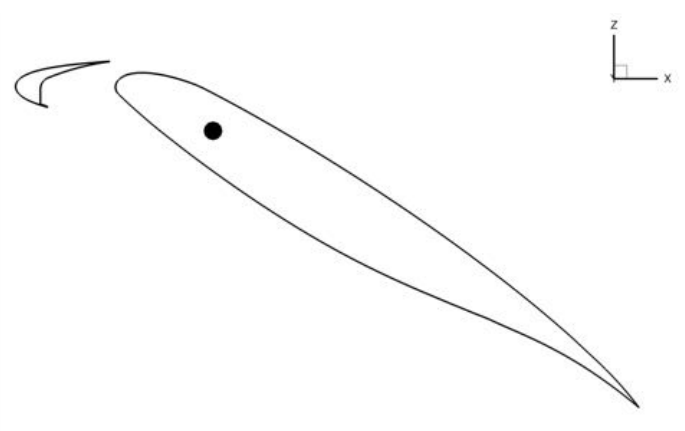

(a)

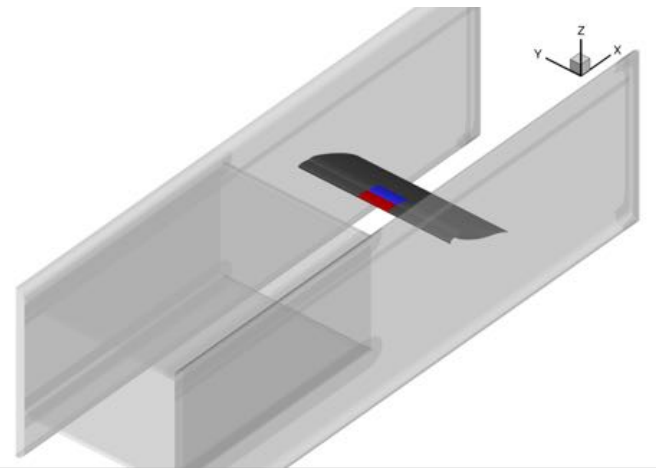

(b)

Figure 2. (a) Profile of the modified (slat) $30 \mathrm{P} 30 \mathrm{~N}$ with flap retracted, slat at 30 degree deployment, and installed at 27 degrees angle of attack. (b) Isometric view of the installed 2-element (flap retracted) high-lift model with a transparent image of the nozzle and side-walls. 


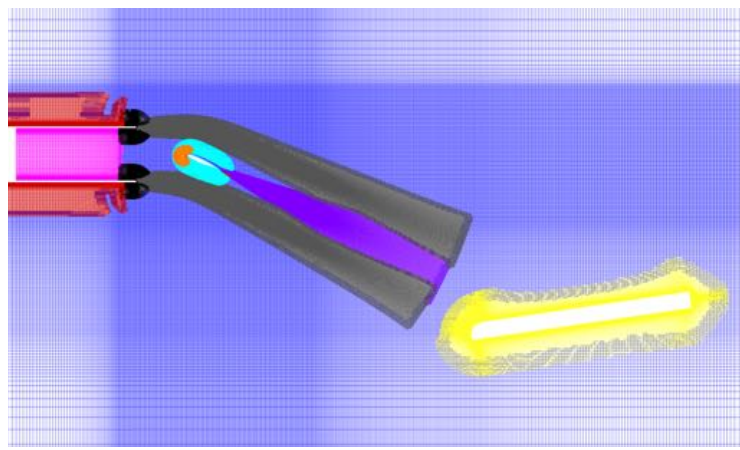

(a)

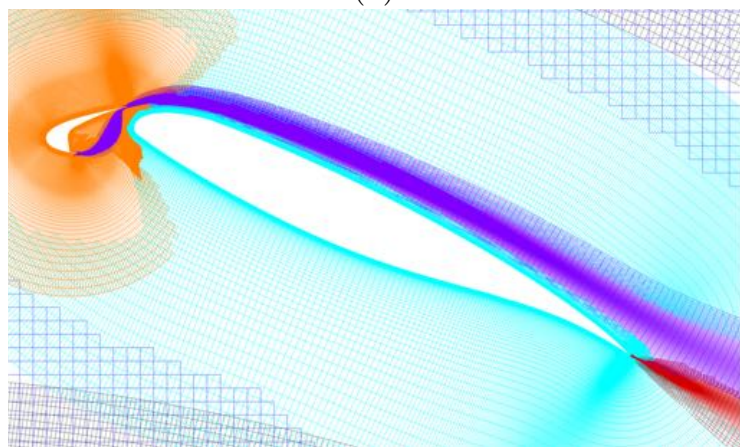

(c)

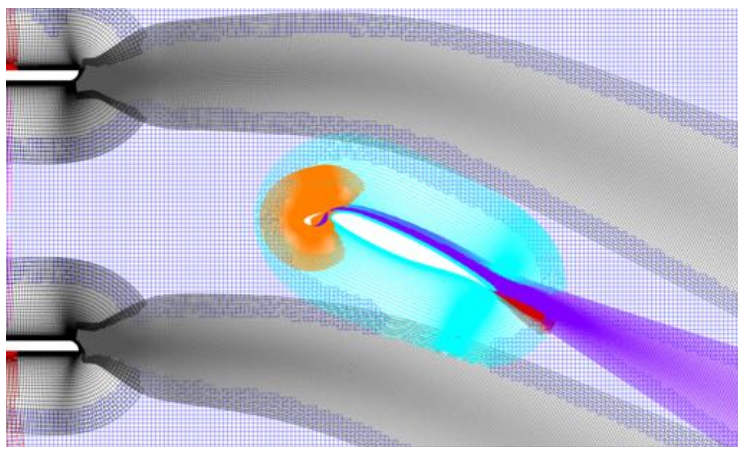

(b)

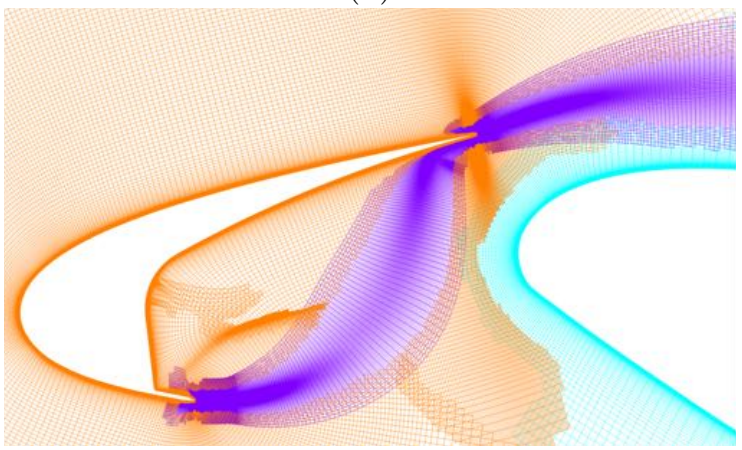

(d)

Figure 3. Structured overset grid system for the installed high-lift model: (a) view of nozzle, slat, main element, and collector plate, (b) illustration of the nozzle shear layer grids which are designed to follow the streamlines of the open-jet which are deflected as they pass around the high-lift model, (c) plot of the slat and main element grids which include specially designed wake grids, and (d) close-up view of the overset grid system in the slat cove and slat wake regions. 


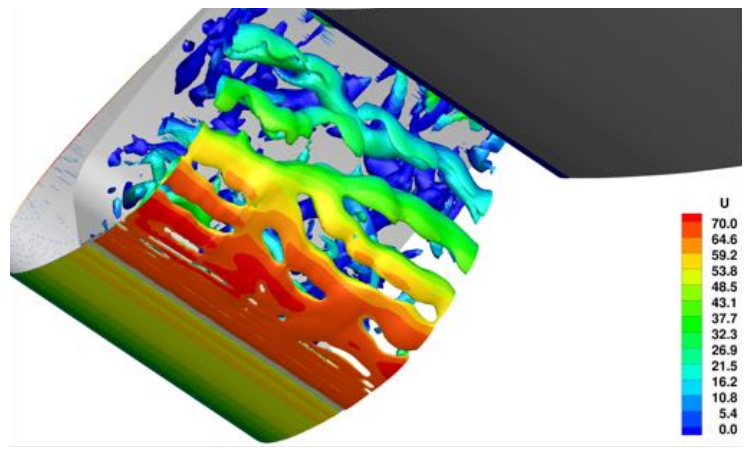

(a)

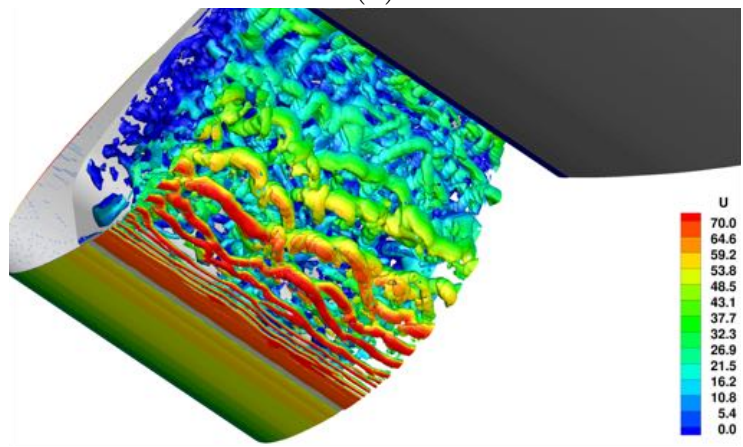

(c)

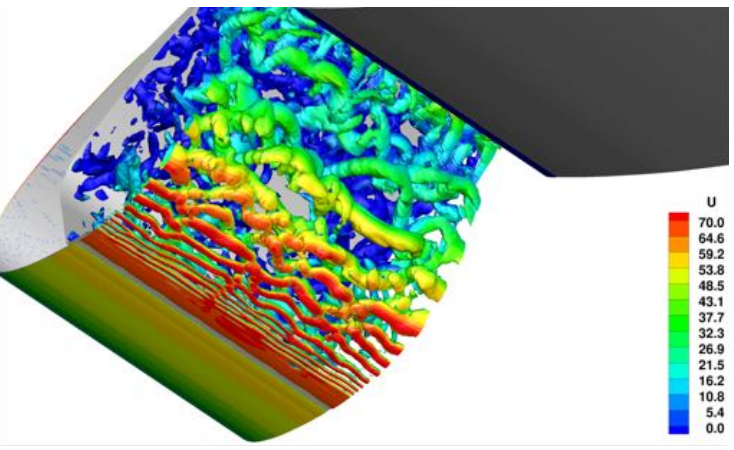

(b)

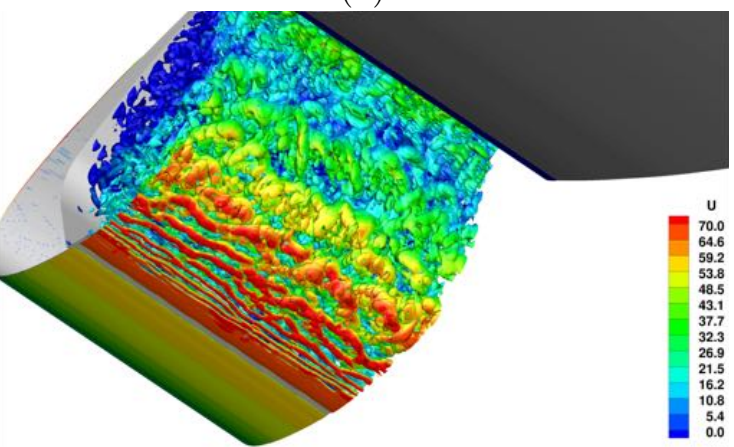

(d)

Figure 4. Improvements to the resolution capacity of the LAVA structured overset grid solver are illustrated by iso-contours of Q-criteria colored by stream-wise velocity using the medium span-wise grid resolution. (a) Original discretization using 5th order ZWENO interpolation to half-points and standard DDES length scale-definition, (b) modified length-scale definition allowing two-dimensional Kelvin-Helmholtz instability to be captured, (c) use of optimal weights in the interpolation procedure which remains stable for this sub-sonic flow condition, and (d) increase to 7 th order interpolation in span-wise direction and use of the blended central/upwind biased interpolation based on local Mach number.

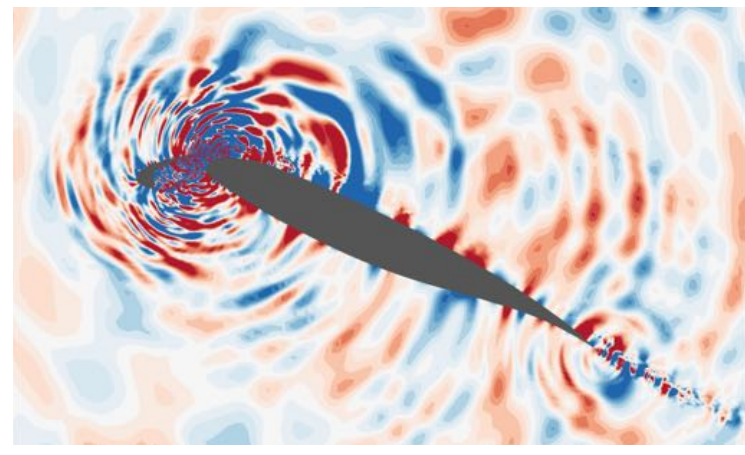

(a)

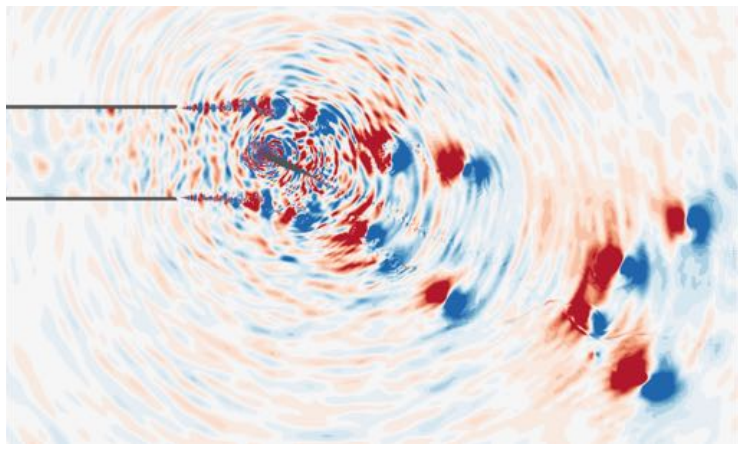

(b)

Figure 5. Contour plot of time-derivative of pressure (a) close-up view showing the noise generated from the slat cove and trailing edge, and (b) mid-field view illustrating the acoustic radiation pattern and its interaction with the noise generated from the open-jet nozzle shear layers 


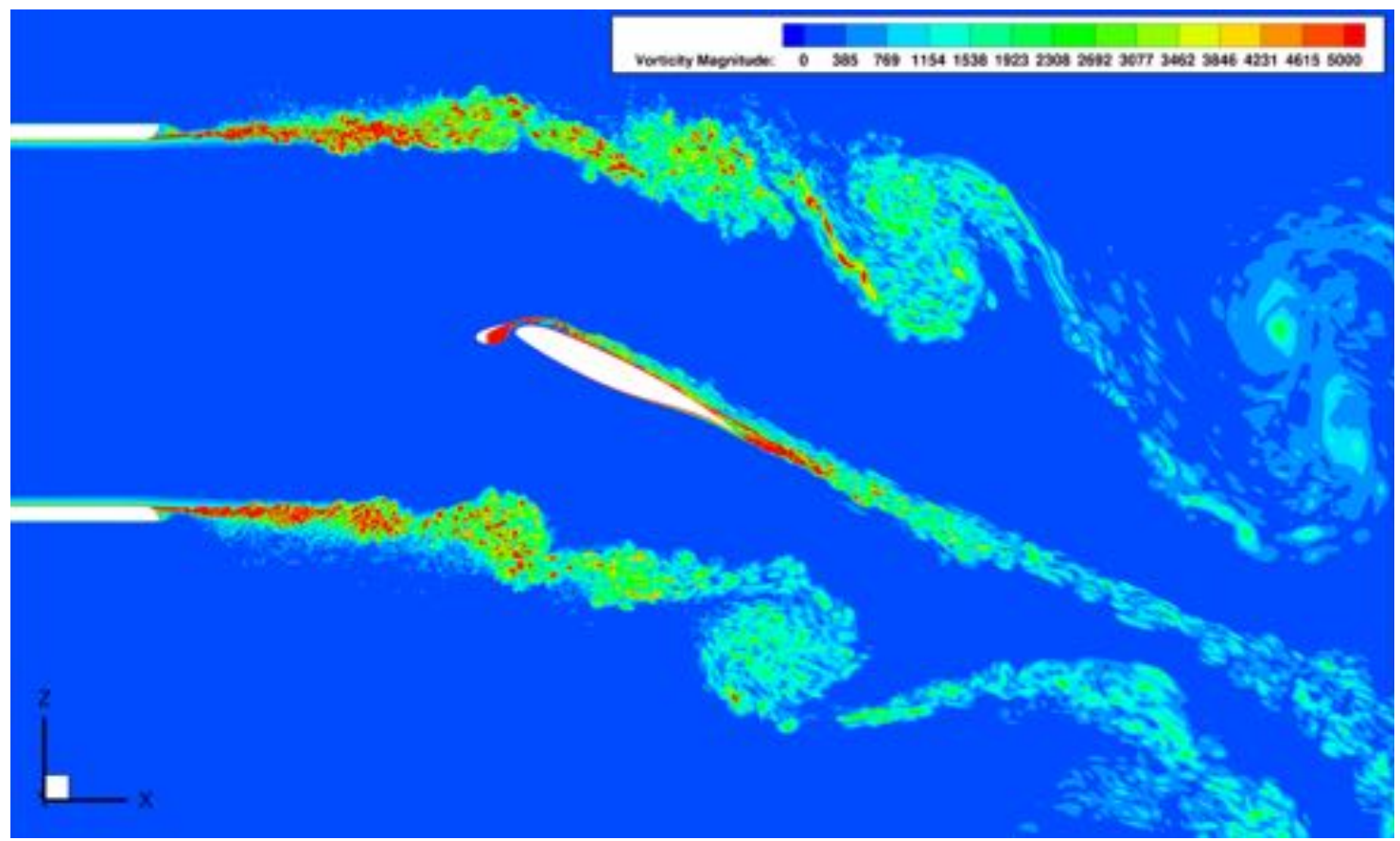

Figure 6. Contour plot of vorticity magnitude on a two-dimensional cutting plane through the center of the domain showing large scale vortex structures comprised of smaller scale turbulent structures emanating from the slat, the trailing edge of the main element, and the exit of the open-jet nozzle.

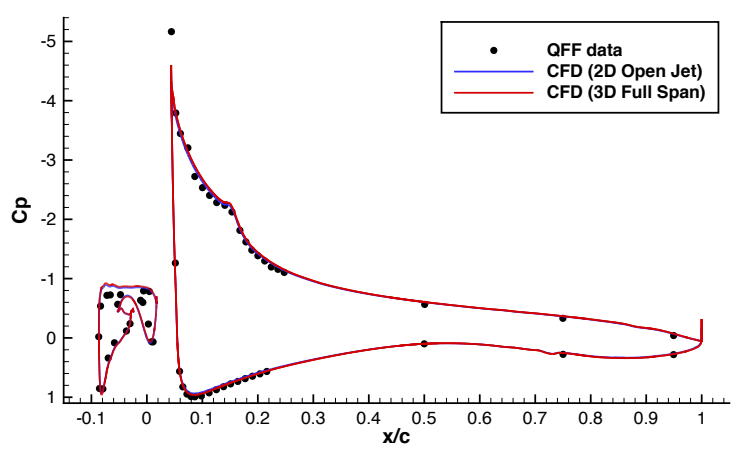

(a)

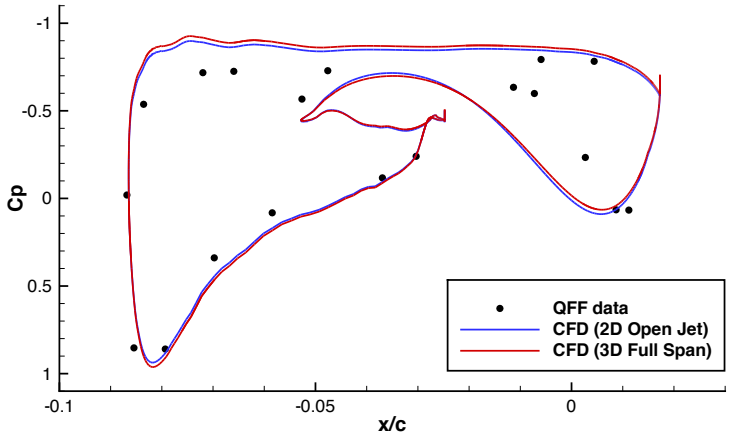

(b)

Figure 7. Comparison of time-averaged coefficient of pressure (Cp) showing the experimental results with the twodimensional constant span open-jet configuration (simulation is still three-dimensional) and an unsteady full span three-dimensional RANS calculation (including side walls). (a) Full chord and (b) a close-up of the slat region. 


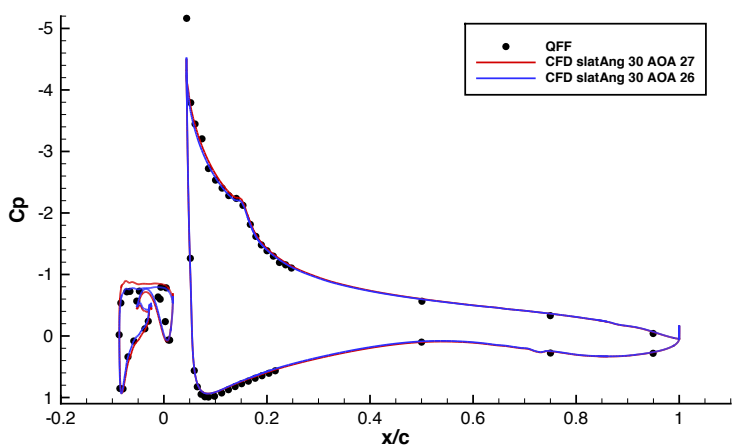

(a)

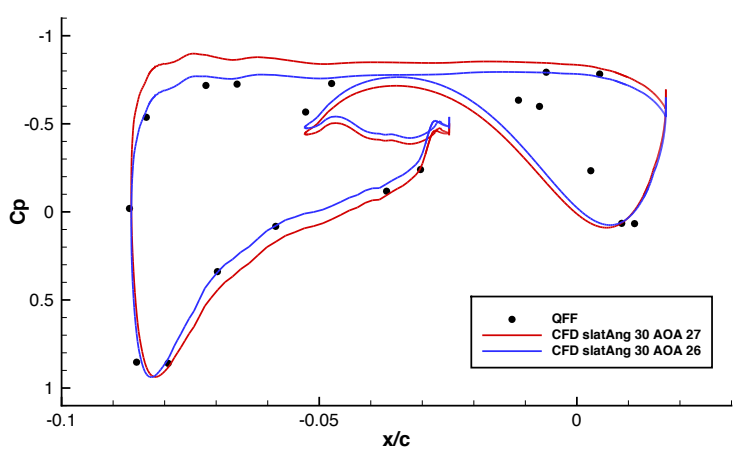

(b)

Figure 8. Plot of time-averaged Cp assessing an angle of attack correction to account for possible chamber circulation effects or installation measurement errors. (a) Full chord and (b) a close-up of the slat region.

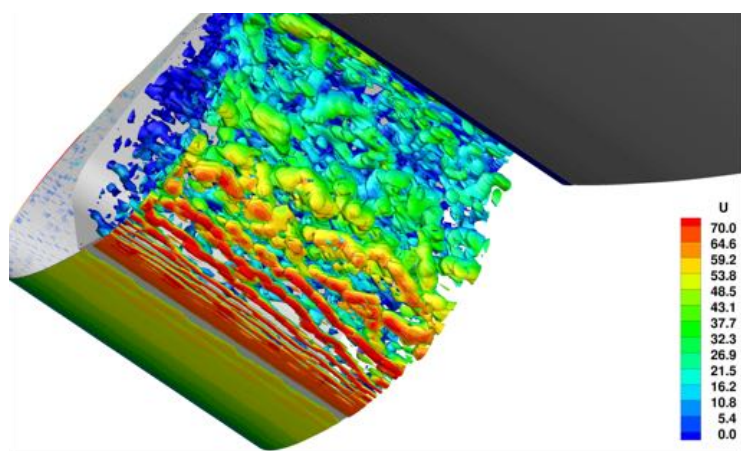

(a)

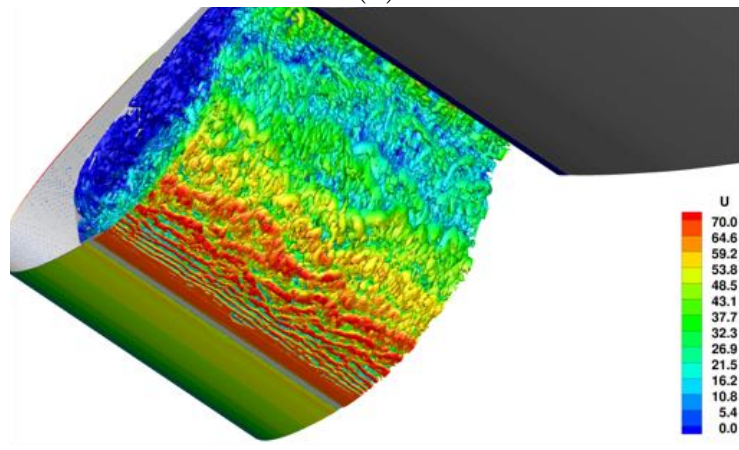

(c)

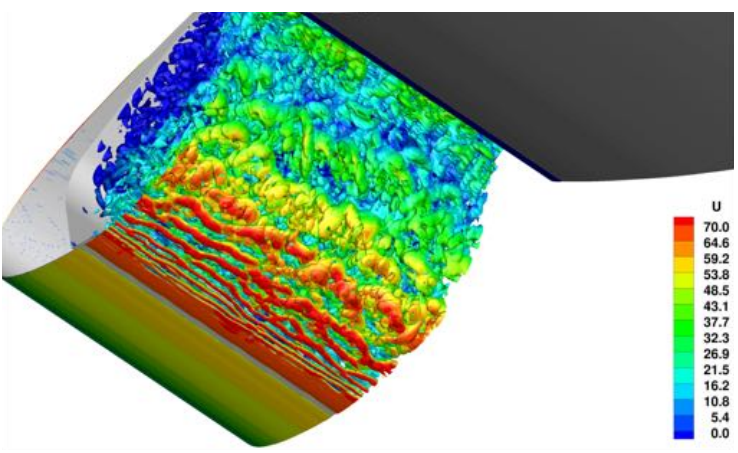

(b)
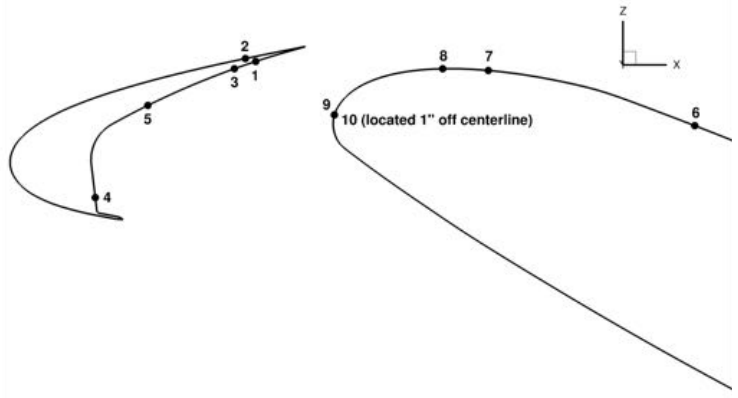

(d)

Figure 9. (a-c) Iso-contour plot of Q-criteria colored by stream-wise velocity using coarse, medium, and fine span-wise resolution grids. (d) Diagram showing Kulite dynamic pressure transducer locations on the slat (transducers 1-5) and the main element (transducers 6-10). Note that transducers 9 and 10 are located at the same location on the airfoil profile, but transducer 9 is on the center-span of the model while transducer 10 is positioned one inch off the centerline. 


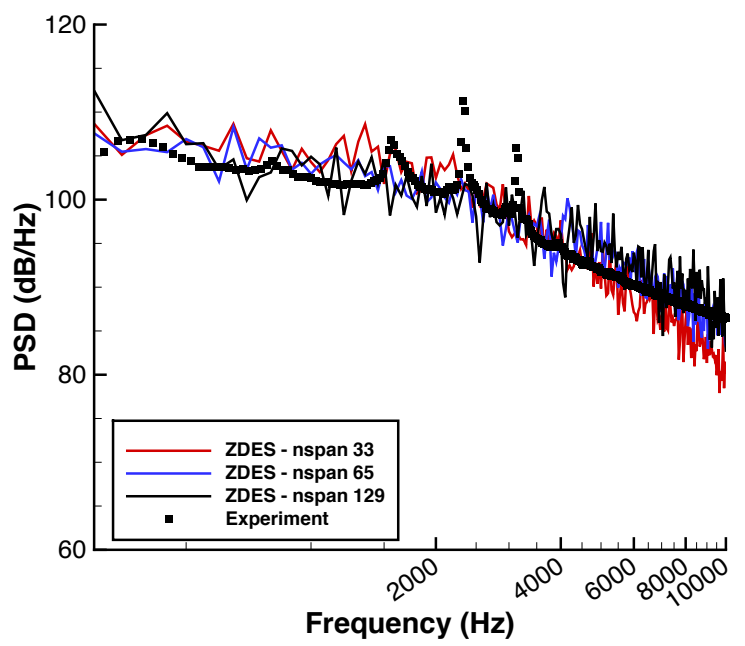

(a) Dynamic Pressure Transducer 1

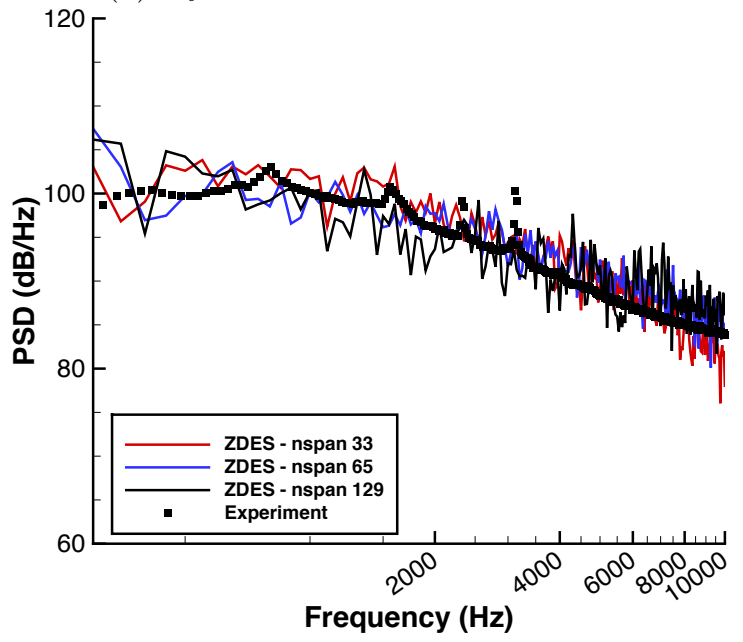

(c) Dynamic Pressure Transducer 3

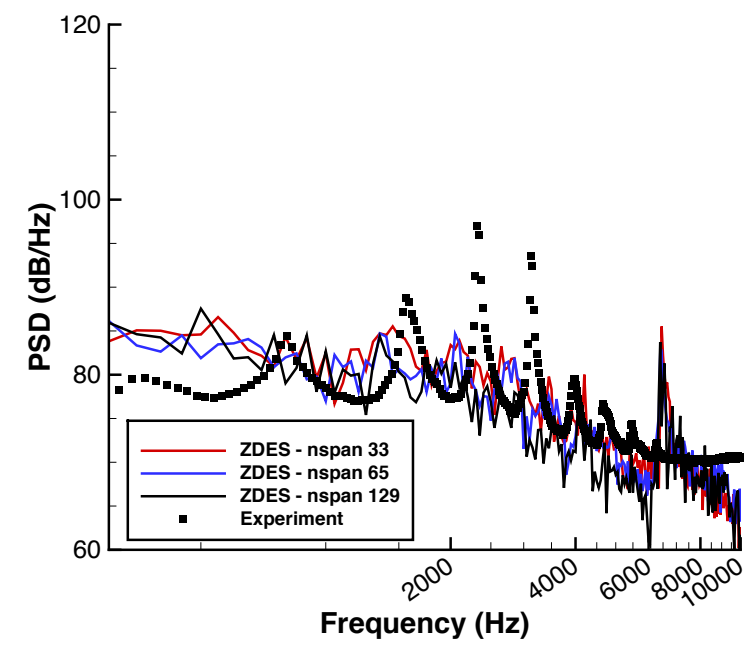

(b) Dynamic Pressure Transducer 2

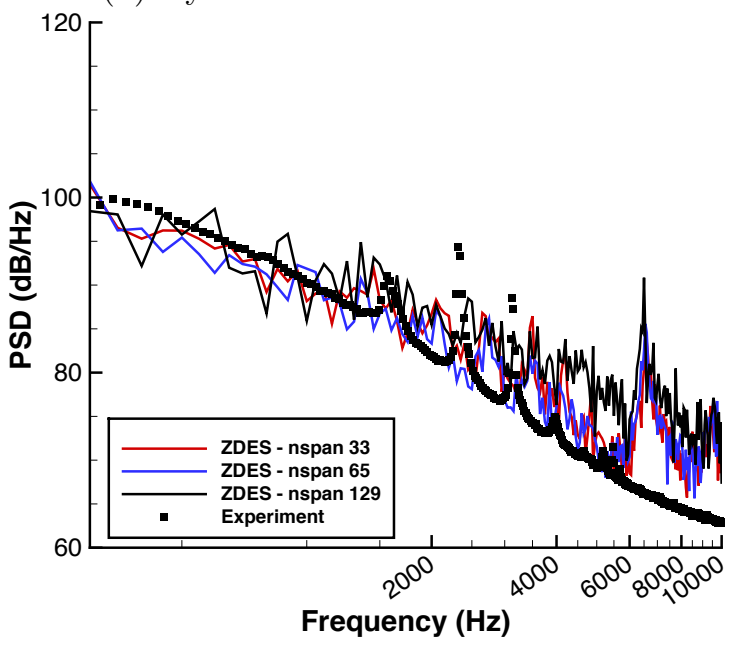

(d) Dynamic Pressure Transducer 4

Figure 10. Power Spectral Density (PSD) spectrum on the slat using different span-wise grid resolutions compared to experimental data for dynamic pressure transducers 1 (a), 2 (b), 3 (c), and 4 (d). 


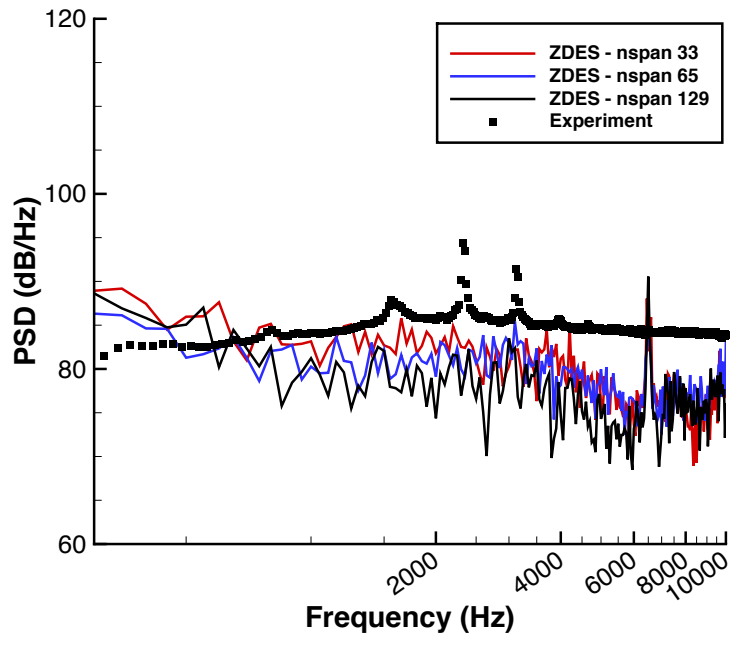

(a) Dynamic Pressure Transducer 6

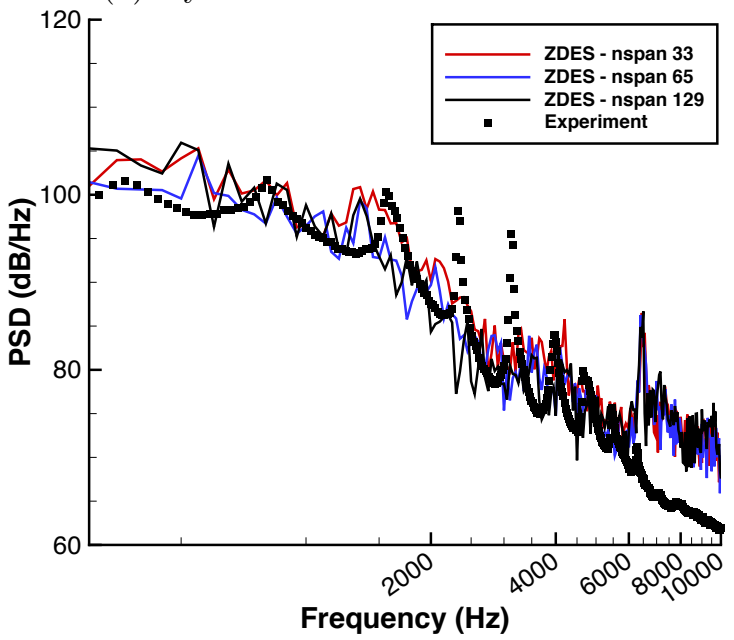

(c) Dynamic Pressure Transducer 9

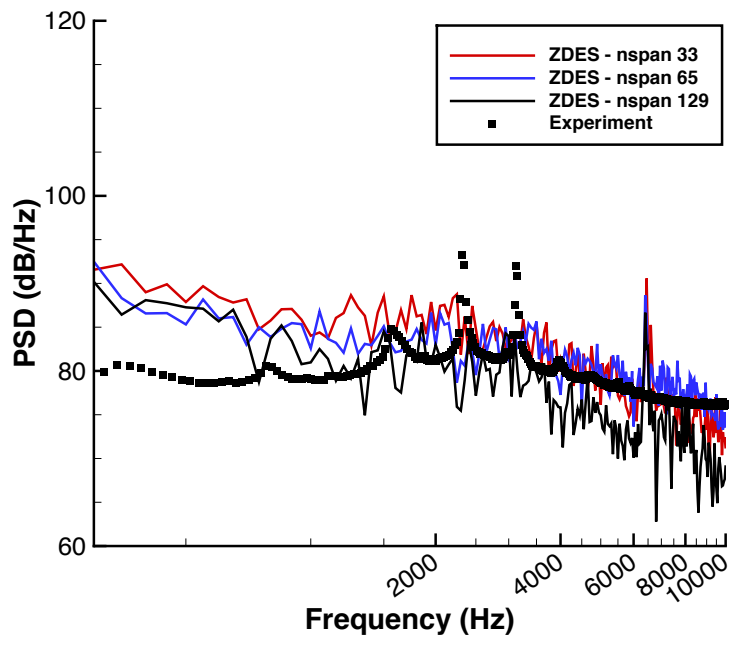

(b) Dynamic Pressure Transducer 7

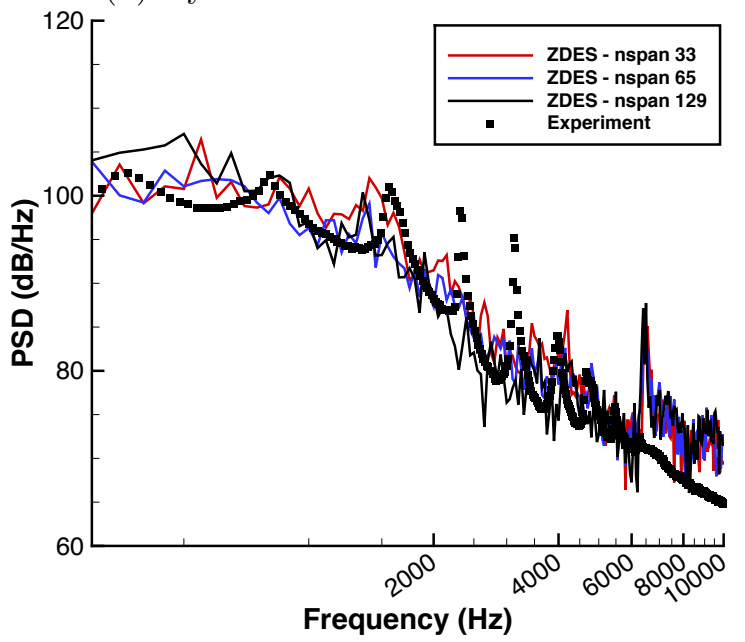

(d) Dynamic Pressure Transducer 10

Figure 11. Power Spectral Density (PSD) spectrum on the main-element using different span-wise grid resolutions compared to experimental data for dynamic pressure transducers 6 (a), 7 (b), 9 (c), and 10 (d).

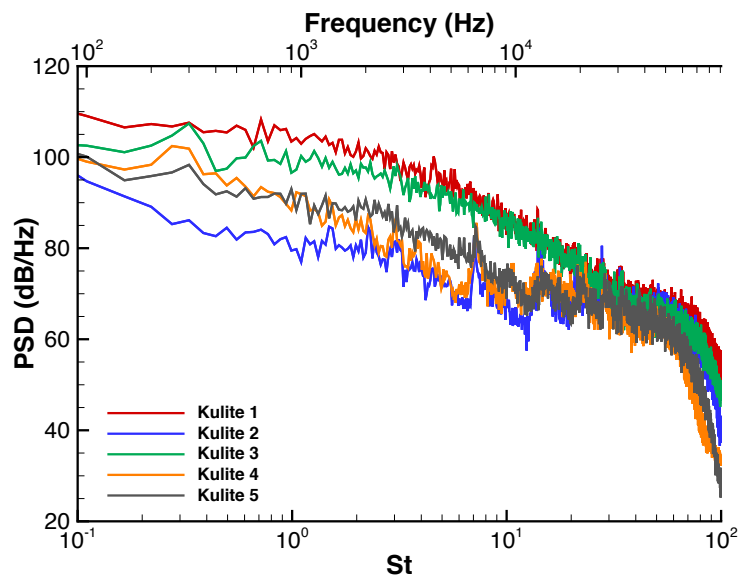

(a)

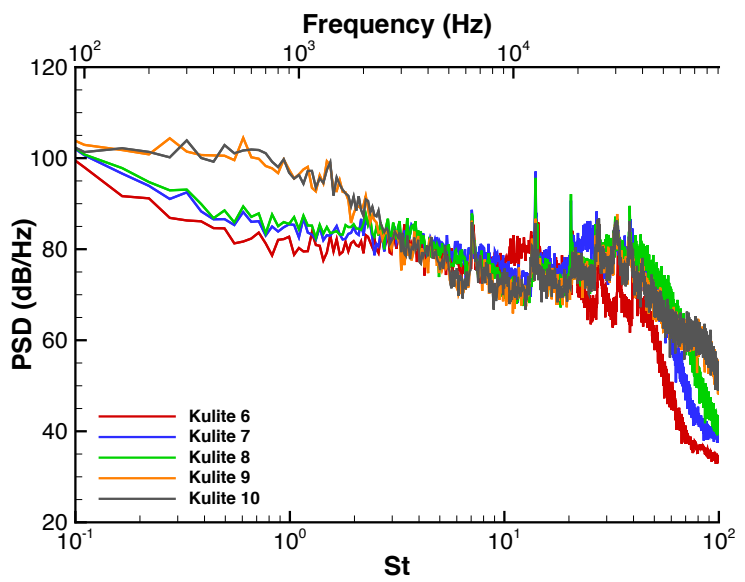

(b)

Figure 12. Plot of Power Spectral Density (PSD) spectrum for (a) dynamic pressure transducers located on the slat and (b) dynamic pressure transducers located on the main element using the medium span-wise resolution mesh 